\title{
Grouping of Frankia strains on the basis of susceptibility to antibiotics, pigment production and host specificity
}

\author{
Svetlana V. Dobritsa† \\ Tel: + 7967732684 . Fax: +7959233602. \\ e-mail: dobritsa $@$ ibpm.serpukhov.su
}

Department All-Russian Collection of

Microorganisms, Institute of Biochemistry and Physiology of Microorganisms, Russian Academy of Sciences, Pushchino, Moscow Region, 142292, Russia

\begin{abstract}
Thirty-nine selected Frankia strains belonging to different genomic species were clustered on the basis of their in vitro susceptibility to 17 antibiotics, pigment production and ability to nodulate plants of the genus Alnus and/or the family Elaeagnaceae, or the family Casuarinaceae. The majority of the strains studied fell into three cluster groups, A, E and C, corresponding to the three host-specificity groups, Alnus, Elaeagnus and Casuarina. Within the groups, eight composite clusters, consisting of at least two strains, and five single-member clusters were recovered at the 0.74 distance level, in good agreement with levels of genetic relatedness between the strains. In addition, five strains were recovered as single-member clusters not in the cluster groups, four of them representing single-member genospecies and one strain not assigned to any known genospecies. The concordance between the phenotypic clusters and the genospecies described previously shows that the grouping may reflect the taxonomic structure of the genus Frankia. For some clusters, differentiating phenotypic characters were found which may be useful for species definition.
\end{abstract}

Keywords: Frankia, phenotypic clusters, differentiating characters

\section{INTRODUCTION}

Actinomycetes of the genus Frankia are nitrogenfixing root nodule symbionts of diverse non-legume dicotyledonous plants (for a review, see Benson \& Silvester, 1993). The first attempt to classify these micro-organisms was undertaken in the absence of pure cultures. In 1970, Becking described 10 species of Frankia, based on the source plant and the morphology of endophytes within root nodules. Since the first isolation of a Frankia strain in pure culture (Callaham et al., 1978), a large number of strains have been isolated and studied in vitro and it has become evident that the previous taxonomic system (Becking, 1970) is not correct. Numerous attempts have been made to divide Frankia isolates into groups on the basis of their morphology, physiology, cell chemistry, etc. (for a review, see Lechevalier, 1994) and some potentially useful taxonomic characters have been revealed. However, the relationships of the resulting groups have been difficult to discern because the strains studied

†Present address: Department of Botany, 437 Hesler Biology Building, University of Tennessee, Knoxville, TN 37996-1100, USA. were often few and different from study to study. Host plant responses have been used to group Frankia strains into three major host-specificity groups (Normand \& Lalonde, 1986; Baker, 1987; Torrey, 1990). The Alnus group includes strains able to nodulate Alnus (Betulaceae), Myrica and Comptonia (Myricaceae). The Elaeagnus group includes strains infective on Elaeagnus, Hippophaë, Shepherdia (Elaeagnaceae) and Colletia (Rhamnaceae). The Casuarina group includes strains infective on Casuarina and Allocasuarina (Casuarinaceae). Lalonde and coworkers proposed a redefinition of two of Becking's species, Frankia alni and 'Frankia elaeagni', to accommodate strains according to their host-specificity group, Alnus or Elaeagnus, respectively (Lalonde et al., 1988). In addition, $F$. alni has been proposed to be divided into two subspecies, pommerii and vandijkii, based on the ability of strains to sporulate in root nodules.

Since then, a total of 19 genomic species have been delineated among Frankia strains on the basis of their DNA-DNA relatedness (Fernandez et al., 1989; Akimov \& Dobritsa, 1992; Lumini et al., 1996), which is currently believed to be the best method to assess 
relationships among strains at the species level (Wayne et al., 1987; Ursing et al., 1995; Vandamme et al., 1996). These include seven genospecies among Alnuscompatible strains, 11 genospecies among Elaeagnaceae-compatible strains and one genospecies among Casuarinaceae-compatible strains. These studies have shown that host specificity is indeed a strong criterion which allows division of Frankia strains into large groups; however, these are of supraspecific, rather than specific, rank. Thus, additional species of Frankia need to be described. The genospecies delineated have not been described as nomenclatural species, since phenotypic characters have not been available for their differentiation, as proposed by Wayne et al. (1987).

A search for differentiating taxonomic characters is exceptionally laborious and time-consuming in the case of frankiae. Frankia strains are morphologically similar and cannot be differentiated by this criterion. Physiological characters are more diverse. However, they have been studied on a limited basis and only in a few strains. Frankia strains grow very slowly, with generation times up to several days (Akkermans et al., 1992) and are routinely cultivated in liquid media, with only single strains being able to grow on the agar surface. Thus, a very high level of sterility in experiments is required, all the more so since homogenization of mycelium and high inoculum density are needed to obtain subcultures (Baker, 1990). The latter circumstance, moreover, does not practically allow a reliable visual estimation of growth. As a consequence, the ability to grow on various $\mathrm{C}$ and $\mathrm{N}$ sources, vitamin requirements, effects of growth inhibitors, $\mathrm{pH}$, temperature, water potential, etc., have been typically determined on the basis of biomass increase. This has been estimated, more or less accurately, by measuring dry weight, total organic carbon, total protein, total ATP, optical density of sonicated cultures and packed cell volume, etc. (e.g. Blom et al., 1980; Burggraaf \& Shipton, 1982; Shipton \& Burggraaf, 1982a, b; Vogel \& Dawson, 1986; Dawson \& Gibson, 1987; FaureRaynaud et al., 1990). Altogether, these difficulties preclude extensive physiological searches for differentiating characters, as well as numerical taxonomic analysis of phenotypic traits which has been proved to be a useful tool for delineating bacterial taxospecies. Therefore, computer groupings of Frankia strains have only been performed on the basis of chemotaxonomic characters, namely, whole-cell sugar composition (StLaurent et al., 1987) and fatty acid composition (Simon et al., 1989; Mirza et al., 1991), as well as of isoenzyme patterns (Gardes et al., 1987). However, these studies have included only a few, if any, strains studied by DNA-DNA hybridization and this considerably diminishes the taxonomic value of the results obtained. The above-mentioned problems and others have even culminated in a point of view that the progress in classification of difficult-to-grow Frankia spp. is only possible through molecular, not phenotypic, studies (Lechevalier, 1994).

We studied 39 Frankia strains with the aim of searching for easily determined phenotypic characters of possible taxonomic value. Thirty-four strains represented 16 of the 19 genospecies described so far (Fernandez et al., 1989; Akimov \& Dobritsa, 1992; Lumini et al., 1996). Most of the other strains ( 4 of 5) have also been studied by DNA-DNA hybridization (Fernandez et al., 1989) but have not been assigned to genospecies due to incomplete data. The strains were tested for their in vitro susceptibility to 18 antibiotics and for pigment production, and these data, combined with previously reported data on host specificity, were used for computer grouping of the strains. This approach resulted in phenotypic clusters corroborating the genospecies delineated within the genus Frankia and in revealing differentiating characters for some clusters.

\section{METHODS}

Strains. The Frankia strains used and their sources are listed in Table 1.

Growth conditions. The strains were routinely grown in submerged liquid cultures at $28^{\circ} \mathrm{C}$ for 3 weeks without agitation. The growth media numbered in Table 1 are as follows: 1, 0.5 $\times$ QMod/Tween (Blom et al., 1980); $2, \mathrm{P}+\mathrm{N}$ (Hafeez et al., 1984) without biotin; 3, FTW (Simonet et al., 1985); 4, QMod without Tween 80; 5, BAP (Murry et al., 1984); 6, OS-1 (Dobritsa \& Stupar, 1989); 7, FTW without Tween 80 . Pigment production by all strains was observed in $\mathrm{P}+\mathrm{N}$ medium, as well as in the medium in which the strain grew best (Table 1), if different from $\mathrm{P}+\mathrm{N}$. Pigments were categorized into one of four colour groups (Table 2).

Antibiotic susceptibility tests. For antibiotic susceptibility tests, inocula were prepared from $15 \mathrm{ml}$ cultures maintained in the medium in which they grew best. They were concentrated by centrifugation $(3000 \mathrm{~g}, 10 \mathrm{~min})$, washed twice with distilled water, resuspended in $5 \mathrm{ml} \mathrm{P}+\mathrm{N}$ medium and inoculated into $200 \mathrm{ml} \mathrm{P}+\mathrm{N}$ medium in $500 \mathrm{ml}$ flasks. After 3 weeks incubation, the mycelium (about $0.1-0.15 \mathrm{ml}$ cell packed volume $\mathrm{ml}^{-1}$ ) was homogenized in the culture medium with a teflon-pestle glass homogenizer and mixed with an equal volume of $\mathrm{P}+\mathrm{N}$ medium containing $1.5 \%$ $(\mathrm{w} / \mathrm{v})$ Bacto-agar (Difco) which had been melted and maintained at $45{ }^{\circ} \mathrm{C}$. The cell suspension containing $0.75 \%$ agar was used to overlay $\mathrm{P}+\mathrm{N}$ plates containing $1.5 \%$ Bacto-agar $(3 \mathrm{ml}$ per $90 \mathrm{~mm}$ plate or $4.5 \mathrm{ml}$ per $110 \mathrm{~mm}$ plate). After the top layer had solidified, antibiotic disks (Difco) were placed aseptically onto the agar in the centre (one disk per plate). The dishes were sealed with Parafilm and incubated at $28^{\circ} \mathrm{C}$. After $14 \mathrm{~d}$ incubation, growth of strains was compared with that on control plates without antibiotics and inhibition zone diameters around the disks were measured. Disks containing ampicillin ( $10 \mu \mathrm{g}$ per disk), bacitracin $(10 \mathrm{U})$, carbenicillin $(100 \mu \mathrm{g})$, chloramphenicol $(30 \mu \mathrm{g})$, clindamycin $(2 \mu \mathrm{g})$, erythromycin $(15 \mu \mathrm{g})$, gentamicin $(10 \mu \mathrm{g})$, kanamycin $(30 \mu \mathrm{g})$, lincomycin $(2 \mu \mathrm{g})$, nalidixic acid $(30 \mu \mathrm{g})$, neomycin $(30 \mu \mathrm{g})$, novobiocin $(30 \mu \mathrm{g})$, penicillin $\mathrm{G}(10 \mathrm{U})$, polymyxin $\mathrm{B}(300 \mathrm{U})$, rifampicin $(5 \mu \mathrm{g})$, streptomycin $(10 \mu \mathrm{g})$, tetracycline $(30 \mu \mathrm{g})$ and vancomycin $(30 \mu \mathrm{g})$ were used.

Coding of data. There were 21 rows (characters) and 39 columns (strains) in the final data matrix. Seventeen characters were derived from the antibiotic susceptibility data (excluding the invariable character of susceptibility to nalidixic acid). The mean inhibition zone diameter ( $\mathrm{mm}$ ) for 
Table 1. Frankia strains used and their sources

\begin{tabular}{|c|c|c|c|c|}
\hline Strain & Original host & Geographic origin & Reference & Growth medium* \\
\hline \multicolumn{5}{|c|}{ Alnus host-compatibility group } \\
\hline CpIl & Comptonia peregrina & Massachusetts, USA & Callaham et al. (1978) & 1 \\
\hline Arl3 & Alnus rubra & Oregon, USA & Berry \& Torrey (1979) & 1 \\
\hline AvcIl & Alnus viridis subsp. crispa & Ontario, Canada & Baker et al. (1979) & 1 \\
\hline AvcI1.R1 & Alnus glutinosa & The Netherlands & Akimov \& Dobritsa (1992) & 1 \\
\hline ArI4 & Alnus rubra & Washington, USA & Baker et al. (1981) & 1 \\
\hline Airl1 & Alnus incana subsp. rugosa & Vermont, USA & Lechevalier et al. (1983) & 1 \\
\hline An2.1 & Alnus nitida & Swat, Pakistan & Hafeez et al. (1984) & 2 \\
\hline An2.24 & Alnus nitida & Swat, Pakistan & Hafeez et al. (1984) & 2 \\
\hline Ailc & Alnus incana & Hämeenlinna, Finland & Weber et al. (1988) & 3 \\
\hline Ail5a & Alnus incana & Hämeenlinna, Finland & Weber et al. (1988) & 3 \\
\hline Ag9b & Alnus glutinosa & Hämeenlinna, Finland & Weber et al. (1988) & 3 \\
\hline Ai6b & Alnus incana & Helsinki, Finland & Weber et al. (1988) & 3 \\
\hline AgI5 & Alnus glutinosa & Oostvoorne, The Netherlands & Hahn et al. (1989) & 2 \\
\hline $\mathrm{ARgP} 5^{A(i}$ & Alnus rugosa & Québec, Canada & Normand \& Lalonde (1982) & 2 \\
\hline $\mathrm{Mg} 60_{2}{ }^{\mathrm{Ag}}$ & Alnus glutinosa & Landes, France & Fernandez et al. (1989) & 3 \\
\hline AirI2 & Alnus incana subsp. rugosa & Vermont, USA & Lechevalier et al. (1983) & 4 \\
\hline \multicolumn{5}{|c|}{ Elaeagnus host-compatibility group $\dagger$} \\
\hline E1 & Elaeagnus hybrid & Camaldoli, Italy & Bosco et al. (1992) & 5 \\
\hline E3 & Elaeagnus hybrid & Camaldoli, Italy & Bosco et al. (1992) & 5 \\
\hline EanII57 & Elaeagnus hybrid & Camaldoli, Italy & Margheri et al. (1985) & 5 \\
\hline Ccl.17 & Colletia cruciata & The Netherlands & Hafeez et al. (1984) & 2 \\
\hline S14 & Shepherdia argentea & Samara, Russia & Dobritsa \& Stupar (1989) & 6 \\
\hline $\mathrm{S} 15$ & Shepherdia argentea & Samara, Russia & Dobritsa \& Stupar (1989) & 6 \\
\hline $\mathrm{S} 13$ & Shepherdia argentea & Samara, Russia & Dobritsa \& Stupar (1989) & 6 \\
\hline Hr5-o & Hippophaë rhamnoides & Pushchino, Russia & Dobritsa et al. (1990) & 2 \\
\hline $\begin{array}{l}\text { ORS } \\
060501\end{array}$ & Colletia spinosissima & Rio Tercero, Argentina & Gauthier et al. (1984) & 5 \\
\hline $\mathrm{EAN} 1_{\text {pe: }}$ & Elaeagnus angustifolia & Ohio, USA & Lalonde et al. (1981) & 7 \\
\hline GFN14 & Hippophaë rhamnoides & China & Fernandez et al. (1989) & 5 \\
\hline K1510 & Elaeagnus griffithii & Yunnan, China & Du \& Baker (1992) & 5 \\
\hline D11 & Casuarina equisetifolia & Dakar, Senegal & Gauthier et al. (1981) & 5 \\
\hline $\mathrm{Eal}_{12}$ & Elaeagnus angustifolia & Ecully, France & Fernandez et al. (1989) & 7 \\
\hline EaI11 & Elaeagnus argentea & Pushchino, Russia & Sharaya et al. (1985) & 6 \\
\hline D-EaI11: & Elaeagnus argentea & Pushchino, Russia & S. V. Dobritsa, unpublished data & 2 \\
\hline D-EaI10 + & Elaeagnus argentea & Pushchino, Russia & S. V. Dobritsa, unpublished data & 2 \\
\hline $\mathrm{Ea}_{50}$ & Elaeagnus angustifolia & Mions, France & Fernandez et al. (1989) & 7 \\
\hline HRN18a & Hippophaë rhamnoides & Alps, France & Moiroud \& Faure-Raynaud (1983) & 7 \\
\hline EuIlb & Elaeagnus umbellata & Massachusetts, USA & Baker et al. (1979) & 1 \\
\hline \multicolumn{5}{|c|}{ Casuarina host-compatibility group } \\
\hline $\mathrm{CeD}$ & Casuarina equisetifolia & Dakar, Senegal & Diem \& Dommergues (1983) & 5 \\
\hline K114 & Casuarina equisetifolia & Yunnan, China & Shi et al. (1991) & 5 \\
\hline AllI1 & Allocasuarina lehmaniana & Florida, USA & Zhang \& Torrey (1985) & 5 \\
\hline
\end{tabular}

* Defined in Methods.

† Includes strains infective on both Elaeagnaceae and Alnus.

$\$$ Spontaneous, better-growing derivatives of strains EaI11 and EaI10, respectively.

a given antibiotic tested against a certain strain was determined from several (at least two) independent tests, rounded off to the nearest $\mathrm{mm}$ and the resulting value was used as a quantitative character for the data matrix. When no inhibition zones were observed in all parallel tests and, in fact, mycelial growth could be observed beneath a disk, ' 0 ' was used for coding the mean zone diameter. If small inhibition zones were observed in some and not in other parallel tests for a given antibiotic/strain combination, zone diameters in those tests that showed no inhibition zones were assumed to be equal to $6 \mathrm{~mm}$ (diameter of an antibiotic disk). Pigment production was coded as binary data: 1 for + and \pm and 0 for - and $\mp$ (see Table 2). The qualitative multistate characters of host specificity were coded as three independent characters and each was scored $+(1)$ for the character state shown and $-(0)$ for the alternative. 
Cluster analysis. Computer analyses of the data were performed on NTSYS-pc software (version 1.70; Applied Biostatistics, 1992). Distances between the strains were calculated on the basis of the standardized data set by using the SIMINT program. Clustering was achieved by using the SAHN program with the unweighted mean linkage (UPGMA) algorithm (Sneath \& Sokal, 1973).

\section{RESULTS AND DISCUSSION}

A sound classification depends upon reliable characters which separate taxa having a high phenotypic similarity. In an attempt to find criteria to be used in the taxonomy of the genus Frankia, we looked for tests which are reproducible, easy to perform and read, routine for bacteriology and least affected by subjective interpretation (Bascomb et al., 1973).

\section{Susceptibility to antibiotics}

As the main group of characters, in vitro susceptibilities of the Frankia strains to 18 antibiotics were studied. Antibiotics acting selectively on certain species have been used for taxonomic purposes in some groups of micro-organisms (Gilardi, 1971; Penner \& Preston, 1980; Nikitin et al., 1988; Reva et al., 1995) or for an intraspecific grouping of strains (Scavizzi \& Bronner, 1987). Computer programs have been written for identification of micro-organisms, based exclusively on susceptibility to antibiotics (Petralli et al., 1970; Friedman \& MacLowry, 1973; Sielaff et al., 1976), since the characters are in good agreement with classical taxonomy. Susceptibility to antibiotics has been shown to be useful for classification and identification of certain actinomycete genera as well (Goodfellow \& Orchard, 1974; Gordon \& Barnett, 1977; Tsukamura, 1981, 1982, 1988).

Preliminary visual estimation of growth of our cultures in liquid media containing dilutions of antibiotics did not give reliable results (S. V. Dobritsa, unpublished observations). Therefore, subsequent experiments were performed by using a modified agar diffusion method. The Frankia strains were grown in a thin layer of semisolid agar on the surface of a solid agar medium which resulted in good growth within an acceptable period of time ( 2 weeks) and in clear-cut, visually determinable inhibition zones around antibiotic disks. To standardize experimental conditions, the same lot of each antibiotic disk was tested against all strains, since we found that different lots might give different results. All strains were tested on the same medium $(\mathrm{P}+\mathrm{N}$ agar $)$, even though some grow somewhat better in different media (see Table 1). This simple medium was selected because propionate is the best $\mathrm{C}$ source for most Frankia strains (Akkermans et al., 1992) and, unlike many other Frankia media, it does not contain Tween 80 . In the presence of Tween 80 , susceptibility of Mycobacterium spp. to antibiotics has been shown to become significantly increased (Naik et al., 1989). This is supposed to result from increased cell wall permeability and, hence, diffusion of an antibiotic into cells. In some cases, increased susceptibility of the Frankia strains to antibiotics on media containing Tween 80 has also been observed (S. V. Dobritsa, unpublished data). The susceptibility of each Frankia strain to each antibiotic was tested at least twice. In case of ambiguous or clearly unexpected results, additional tests were performed. In Table 2, mean inhibition zone diameters obtained are presented (deviations did not usually exceed $10 \%$ ).

All of the strains examined displayed resistance to nalidixic acid and this invariable character was excluded from computer analyses. The levels of susceptibility to carbenicillin, novobiocin, tetracycline, vancomycin, streptomycin, ampicillin, erythromycin, kanamycin and penicillin $\mathrm{G}$ varied widely for different strains (diam. of inhibition zones, 0 -94, 89, 85, 79, 75, $72,72,71$ and $65 \mathrm{~mm}$, respectively). Susceptibility to bacitracin, gentamicin, chloramphenicol, neomycin, clindamycin and rifampicin varied to a lesser extent (41-86 and $0-42,33,29,27$ and $27 \mathrm{~mm}$, respectively). Most of the strains studied were resistant to lincomycin and displayed low levels of susceptibility to polymyxin B (0-17 and 8-17 mm, respectively).

The potential value of antibiotic sensitivity tests in the taxonomy of frankiae can be assessed from the data presented. Consistent and marked differences in in vitro antimicrobial sensitivity patterns were observed between strains representing different genospecies, whereas strains belonging to the same genospecies showed, on the whole, more similarity in their susceptibility patterns. For all antibiotics, except nalidixic acid, differences between genospecies were greater than those within individual genospecies. The mean withingenospecies levels of variance ranged from $3.7 \%$ (for gentamicin) to $22.7 \%$ (for lincomycin) of the wholesample levels of variance. The absolute maximum of variance was observed between the strains belonging to genospecies 1 in their levels of susceptibility to erythromycin, but even this did not exceed $78 \%$ of the total variance of the character. Antibiotic sensitivity tests do, therefore, seem to be of value in the subgeneric classification of the genus. In some cases, there is also good correlation between host-specificity group membership and sensitivity to certain antibiotics. Thus, although the sensitivity patterns are generally heterogeneous and often overlapping within and between the Alnus and Elaeagnus groups, one character, susceptibility to novobiocin, shows a significant discriminating potential at the group level. A larger number of antibiotic susceptibility characters differentiate the homogeneous Casuarina group from the other two groups, as discussed below for cluster C1. Susceptibility to some antibiotics may also reflect other forms of variation in frankiae and adaptation to certain environmental conditions. These relationships are important for understanding the place occupied by these species in microbial and plant-microbial associations.

Susceptibility to antibiotics has been studied pre- 
Table 2. Susceptibility to antibiotics, pigment production and host specificity of the Frankia strains studied

\begin{tabular}{|c|c|c|c|c|c|c|c|c|c|c|c|c|c|c|c|c|c|c|c|c|c|c|c|c|c|c|c|}
\hline \multirow[t]{2}{*}{ Strain } & \multirow{2}{*}{$\begin{array}{l}\text { Geno- } \\
\text { species* }\end{array}$} & \multirow[t]{2}{*}{ Clustert } & \multicolumn{18}{|c|}{ Diameter (mm) of inhibition zone with: $:$} & \multicolumn{4}{|c|}{ Pigment production $\S$} & \multicolumn{3}{|c|}{ Infectivity } \\
\hline & & & Am & $\mathbf{B c}$ & $\mathbf{C b}$ & Cl & $\mathrm{Cm}$ & Em & Gm & $\mathbf{K m}$ & $\mathbf{L m}$ & $\mathrm{Na}$ & $\mathbf{N b}$ & $\mathrm{Nm}$ & $\mathbf{P m}$ & Pn & $\mathbf{R m}$ & $\mathrm{Sm}$ & Tc & $\mathrm{Vm}$ & $\begin{array}{l}\text { Pigment- } \\
\text { ation }\end{array}$ & Colour & $\begin{array}{l}\text { Cellular } \\
\text { pigment }\end{array}$ & $\begin{array}{l}\text { Soluble } \\
\text { pigment }\end{array}$ & Alnus & $\begin{array}{c}\text { Elaeag- } \\
\text { naceae }\end{array}$ & $\begin{array}{c}\text { Casuari- } \\
\text { naceae }\end{array}$ \\
\hline \multicolumn{28}{|c|}{ Almus host-compatibility group } \\
\hline Cpll & 1 & $\mathrm{Al}$ & 37 & 47 & 47 & 20 & 0 & 58 & 28 & 37 & 0 & 0 & 0 & 23 & 10 & 25 & 14 & 37 & 14 & 54 & - & - & - & - & + & - & - \\
\hline Arl3 & 1 & $\mathrm{~A} 1$ & 37 & 45 & 47 & 20 & 0 & 58 & 27 & 37 & 0 & 0 & 0 & 22 & 10 & 33 & 14 & 35 & 14 & 52 & - & - & - & - & + & - & - \\
\hline AvcI 1 & 1 & $\mathrm{Al}$ & 37 & 47 & 48 & 18 & 0 & 59 & 28 & 23 & 0 & 0 & 0 & 22 & 12 & 32 & 14 & 44 & 14 & 58 & - & - & - & - & + & - & - \\
\hline Avcl1.R1 & 1 & $\mathrm{~A} 1$ & 37 & 47 & 48 & 18 & 0 & 58 & 28 & 23 & 0 & 0 & 0 & 22 & 12 & 33 & 14 & 43 & 14 & 53 & - & - & - & - & + & - & - \\
\hline Arl4 & 1 & A1 & 37 & 54 & 48 & 18 & 19 & 67 & 28 & 37 & 12 & 0 & 0 & 23 & 12 & 25 & 16 & 39 & 14 & 51 & - & - & - & - & + & - & - \\
\hline Airl1 & 1 & Al & 37 & 54 & 55 & 18 & 19 & 56 & 27 & 37 & 12 & 0 & 0 & 22 & 12 & 30 & 16 & 38 & 18 & 57 & - & - & - & - & + & - & - \\
\hline$A n 2.1$ & 1 & $\mathrm{~A} 2$ & 37 & 54 & 50 & 32 & 0 & 11 & 27 & 34 & 0 & 0 & 0 & 24 & 12 & 32 & 15 & 56 & 19 & 54 & + & $\mathrm{R}$ & + & + & + & - & - \\
\hline An2.24 & 1 & $\mathrm{~A} 2$ & 33 & 54 & 49 & 30 & 0 & 11 & 32 & 33 & 0 & 0 & 13 & 24 & 12 & 32 & 14 & 62 & 19 & 56 & + & $\mathrm{R}$ & + & + & + & - & $\ldots$ \\
\hline Ailc & $m$ & $\mathrm{~A} 3$ & 44 & 47 & 35 & 0 & 0 & 46 & 30 & 46 & 0 & 0 & 0 & 25 & 16 & 20 & 0 & 54 & 13 & 67 & - & - & - & - & + & - & - \\
\hline Ail $5 \mathrm{a}$ & $P 2$ & $\mathrm{~A} 3$ & 45 & 47 & 33 & 0 & 0 & 46 & 30 & 46 & 0 & 0 & 0 & 25 & 16 & 20 & 0 & 54 & 12 & 66 & - & - & - & - & + & - & - \\
\hline $\mathrm{Ag} 9 \mathrm{~b}$ & 12 & $\mathrm{~A} 3$ & 29 & 47 & 33 & 0 & 0 & 46 & 30 & 46 & 0 & 0 & 0 & 25 & 17 & 22 & 0 & 54 & 13 & 65 & - & - & - & - & + & - & - \\
\hline Ai6b & 13 & A4 & 52 & 60 & 36 & 0 & 0 & 53 & 25 & 48 & 0 & 0 & 0 & 34 & 13 & 31 & 20 & 53 & 14 & 52 & - & - & - & - & + & - & - \\
\hline Agl5 & 14 & 1 & 0 & 65 & 0 & 0 & 23 & 58 & 31 & 45 & 11 & 0 & 0 & 20 & 10 & 0 & 13 & 36 & 0 & 48 & $\dddot{+}$ & $\mathrm{Y}$ & $\mp$ & - & + & - & - \\
\hline $\mathrm{ARgP5}^{\mathrm{Al} ;}$ & 3 & 2 & 0 & 48 & 11 & 21 & 12 & 38 & 20 & 18 & 7 & 0 & 0 & 20 & 10 & 0 & 12 & 32 & 12 & 0 & $\mp$ & $\mathrm{Y}$ & $\mp$ & - & + & - & $\ldots$ \\
\hline $\mathrm{Mg} 60)_{2}^{\mathrm{A}(\mathrm{s}}$ & ' & 4 & 70 & 61 & 94 & 21 & 27 & 83 & 34 & 53 & 17 & 0 & 0 & 31 & 16 & 65 & 15 & 60 & 17 & 79 & - & - & - & - & + & - & - \\
\hline Airl2 & $P 5$ & 5 & 0 & 46 & 0 & 22 & 0 & 20 & 25 & 50 & 0 & 0 & 0 & 37 & 16 & 0 & 14 & 75 & 20 & 0 & + & $\mathrm{O} / \mathrm{B}$ & + & - & + & - & - \\
\hline \multicolumn{28}{|c|}{ Elaeagnus host-compatibility group } \\
\hline E1 & 0 & E1 & 35 & 50 & 35 & 24 & 0 & 34 & 25 & 40 & 0 & 0 & 74 & 23 & 12 & 43 & 13 & 46 & 23 & 45 & + & $\mathrm{R}$ & + & - & + & + & $\ldots$ \\
\hline $\mathrm{E} 3$ & 10 & El & 35 & 60 & 35 & 24 & 0 & 34 & 27 & 40 & 0 & 0 & 75 & 25 & 13 & 44 & 15 & 46 & 25 & 63 & + & $\mathrm{R}$ & + & - & + & + & - \\
\hline EanII57 & 0 & El & 35 & 50 & 43 & 27 & 0 & 42 & 27 & 40 & 0 & 0 & 79 & 23 & 15 & 45 & 15 & 42 & 27 & 47 & + & $\mathrm{R}$ & + & - & + & + & - \\
\hline $\mathrm{Ccl} 1.17$ & $P 7$ & El & 24 & 48 & 31 & 23 & 0 & 20 & 24 & 43 & 0 & 0 & 89 & 21 & 11 & 19 & 15 & 40 & 26 & 60 & \pm & $\mathrm{Y}$ & \pm & - & - & + & - \\
\hline $\mathrm{S} 14$ & 18 & E2 & 53 & 46 & 56 & 23 & 15 & 30 & 22 & 42 & 0 & 0 & 12 & 23 & 12 & 35 & 23 & 35 & 85 & 68 & + & $\mathrm{P}$ & - & + & - & + & - \\
\hline S15 & 18 & $\mathrm{E} 2$ & 52 & 46 & 54 & 21 & 13 & 30 & 22 & 42 & 0 & 0 & 12 & 23 & 12 & 35 & 23 & 35 & 85 & 64 & + & $\mathrm{P}$ & $\ldots$ & + & - & + & - \\
\hline $\mathrm{S} 13$ & 18 & E2 & 72 & 46 & 60 & 25 & 17 & 40 & 24 & 35 & 0 & 0 & 12 & 23 & 12 & 38 & 23 & 33 & 85 & 66 & + & $P$ & - & + & - & + & - \\
\hline $\mathrm{Hr} 5-\mathrm{o}$ & 18 & E2 & 41 & 47 & 45 & 22 & 14 & 43 & 24 & 45 & 6 & 0 & 14 & 22 & 13 & 40 & 20 & 60 & 41 & 59 & + & $P$ & + & - & - & + & - \\
\hline $\begin{array}{l}\text { ORS } \\
060501\end{array}$ & ' & E2 & 48 & 45 & 49 & 24 & 16 & 38 & 18 & 38 & 12 & 0 & 10 & 20 & 12 & 36 & 21 & 46 & 55 & 67 & \pm & $Y$ & \pm & - & - & + & - \\
\hline EAN1 ${ }_{w+r}$ & 5 & E3 & 43 & 55 & 35 & 33 & 14 & 32 & 28 & 57 & 0 & 0 & 11 & 25 & 10 & 25 & 20 & 51 & 55 & 50 & + & $\mathrm{R}$ & + & + & - & + & - \\
\hline GFN14 & ' & E4 & 36 & 61 & 48 & 20 & 13 & 34 & 18 & 38 & 9 & 0 & 22 & 18 & 10 & 23 & 15 & 33 & 27 & 50 & + & $\mathrm{R}$ & + & \pm & - & + & - \\
\hline K 1510 & $\therefore D$ & E4 & 33 & 58 & 33 & 25 & 15 & 34 & 15 & 37 & 7 & 0 & 9 & 17 & 10 & 33 & 23 & 29 & 42 & 45 & + & $\mathrm{R}$ & + & + & - & + & - \\
\hline DII & , & E4 & 28 & 52 & 42 & 23 & 0 & 24 & 25 & 31 & 0 & 0 & 19 & 18 & 12 & 27 & 27 & 23 & 46 & 42 & + & $\mathrm{R}$ & + & - & - & + & - \\
\hline $\mathrm{Eal}_{12}$ & 4 & E5 & 28 & 41 & 45 & 32 & 0 & 65 & 23 & 35 & 0 & 0 & 14 & 18 & 11 & 26 & 20 & 47 & 22 & 58 & + & Y & + & - & - & + & - \\
\hline Ealli & 16 & E6 & 63 & 82 & 63 & 18 & 12 & 30 & 25 & 24 & 6 & 0 & 31 & 20 & 14 & 57 & 18 & 38 & 17 & 55 & $\mp$ & $\mathrm{Y}$ & $\mp$ & $\mp$ & + & + & - \\
\hline D-EaI11 & 126 & E6 & 50 & 82 & 63 & 18 & 12 & 30 & 25 & 23 & 7 & 0 & 27 & 20 & 14 & 50 & 20 & 38 & 17 & 55 & \pm & $\mathrm{Y}$ & \pm & - & + & + & - \\
\hline D-EaII0 & 126 & E6 & 57 & 82 & 61 & 16 & 12 & 30 & 25 & 23 & 6 & 0 & 30 & 20 & 14 & 57 & 18 & 38 & 17 & 65 & + & $\mathrm{Y}$ & + & + & - & + & - \\
\hline $\mathrm{Ea} 50_{1}$ & 8 & E7 & 34 & 65 & 56 & 16 & 12 & 42 & 20 & 37 & 11 & 0 & 14 & 19 & 15 & 33 & 9 & 0 & 22 & 50 & + & $\mathrm{R}$ & + & + & - & + & - \\
\hline HRN $18 \mathrm{a}$ & 7 & E8 & 50 & 70 & 36 & 21 & 19 & 24 & 42 & 77 & 0 & 0 & 40 & 19 & 12 & 50 & 17 & 35 & 27 & 74 & + & $\mathrm{Y}$ & + & - & + & + & - \\
\hline Eullb & 19 & 3 & 40 & 86 & 35 & 29 & 17 & 60 & 39 & 49 & 9 & 0 & 13 & 43 & 14 & 32 & 15 & 53 & 76 & 53 & + & $\mathrm{Y}$ & + & + & - & + & - \\
\hline \multicolumn{28}{|c|}{ Casuarina host-compatibility group } \\
\hline $\mathrm{CeD}$ & 9 & $\mathrm{Cl}$ & 0 & 67 & 0 & 27 & 17 & 60 & 0 & 8 & 15 & 0 & 13 & 18 & 9 & 0 & 0 & 30 & 32 & 62 & $\mp$ & Y & $\mp$ & - & - & - & + \\
\hline K114 & i & $\mathrm{Cl}$ & 0 & 67 & 0 & 20 & 17 & 60 & 0 & 6 & 15 & 0 & 13 & 14 & 9 & 0 & 0 & 30 & 32 & 58 & $\mp$ & Y & $F$ & - & - & - & + \\
\hline All1 1 & y & $\mathrm{Cl}$ & 0 & 67 & 0 & 20 & 21 & 60 & 0 & 16 & 11 & 0 & 13 & 15 & 8 & 0 & 0 & 30 & 50 & 62 & $\mp$ & $\mathrm{Y}$ & $\mp$ & - & - & - & + \\
\hline
\end{tabular}

* Data from Fernandez et al. (1989), Akimov \& Dobritsa (1992), Bosco et al. (1992), Lumini et al. (1996) and S. V. Dobritsa (unpublished data). ?, Available DNA relatedness data do not allow assignment to known or novel genospecies; ND, not determined. $\dagger$ See Fig. 1.

$\$$ Mean values from 2-5 independent tests. Am, Ampicillin; Bc, bacitracin; $\mathrm{Cb}$, carbenicillin; $\mathrm{Cl}$, chloramphenicol; $\mathrm{Cm}$, clindamycin; Em, erythromycin; Gm, gentamicin; Km, kanamycin; Lm, lincomycin; $\mathrm{Na}$, nalidixic acid; $\mathrm{Nb}$, novobiocin; Nm, neomycin; Pm, polymyxin B; Pn, penicillin G; Rm, rifampicin; Sm, streptomycin; Tc, tetracycline; Vm, vancomycin.

$\S+$, Strong pigmentation; \pm , weak reliable pigmentation; $\mp$, weak doubtful pigmentation (solely in old cultures); - , no pigmentation. For details, see Methods. Colour: R, shades of red, reddish brown and chestnut; Y, shades of yellow, pale-yellow, yellowish brown and greyish yellow; $\mathrm{O} / \mathrm{B}$, mycelium orange, spores black; $\mathrm{P}$, pink.

| Data from Gauthier et al. (1981, 1984), Lechevalier et al. (1983), Hafeez et al. (1984), Baker (1987), Nazaret et al. (1989), Dobritsa et al. (1990), Torrey (1990), Bosco et al. (1992), Du \& Baker (1992), Lumini et al. (1996) and S. V. Dobritsa \& S. N. Novik (unpublished data).

- Strain K114 has been studied by DNA-DNA hybridization and assigned to a certain genogroup (Shi et al., 1991) but none of its members have previously been shown to belong to genospecies 9 . It is assigned to genospecies 9 on the basis of author's unpublished data.

viously in some Frankia strains in liquid cultures, regardless of possible taxonomic significance. In particular, two strains have been shown by optical density of sonicated cultures to be resistant to low neomycin concentrations (Faure-Raynaud et al., 1990). Several strains have displayed resistance to high concen- trations of certain antibiotics (Normand \& Lalonde, 1986). However, data from these studies cannot be compared with our results because the strains were grown in rich organic media. In addition, in the latter study growth was estimated visually, which is not reliable in the case of liquid cultures, and a long period 
of incubation $(60 \mathrm{~d})$ might have resulted in inactivation or decomposition of the antibiotics. Antibiotic susceptibility of 11 Frankia isolates from the Rhamnaceae has been determined (Carú, 1993; Carrasco et al., 1995 ) in liquid BAP medium, which is very similar in its composition to $\mathrm{P}+\mathrm{N}$ medium used in this study. Growth of cultures in the presence of certain antibiotic concentrations $\left(1,5\right.$ or $\left.10 \mu \mathrm{g} \mathrm{ml}^{-1}\right)$ was estimated by total soluble protein after $6-12 \mathrm{~d}$ incubation. The isolates have been shown to be resistant or moderately resistant to nalidixic acid (0-39\% growth inhibition) and susceptible to erythromycin, kanamycin, rifampicin, ampicillin and streptomycin (82-100\% inhibition). Susceptibility to lincomycin, chloramphenicol and penicillin $G$ varied, depending upon the strain and antibiotic concentration, in the ranges between 0 and 93, 68 and 100, and 20 and $100 \%$ inhibition, respectively. The data from these studies are consistent with our results obtained by the agar diffusion method for a different set of Frankia strains. It is interesting that for the control strain, BR, isolated from Casuarina sp., $100 \%$ growth inhibition has been observed with erythromycin, $81 \%$ with penicillin $\mathrm{G}$ and $9 \%$ with ampicillin, and no growth inhibition has been observed with kanamycin, chloramphenicol, nalidixic acid and rifampicin at $5 \mu \mathrm{g} \mathrm{ml}^{-1}$. This closely corresponds to the susceptibility patterns obtained in this study for three other Casuarinaceae-specific isolates (Table 2).

\section{Pigment production}

The capability of the Frankia strains to produce pigments was used as an additional character in this study (Table 2). Some members of the genus Frankia are known to produce pigments. There are strains producing red, yellow, orange, pink, brown, greenish and black pigments (Lechevalier et al., 1982). It has been supposed that this variability may be a useful taxonomic character at the species level (Benson \& Silvester, 1993) and production of pigments of certain colours has been used as a character, amongst others, for computer identification of bacteria (Bascomb et al., 1973). Both susceptibility to antibiotics and pigment production have been used as characters in an extensive numerical taxonomic study of actinomycetes of the genus Streptomyces and related genera (Williams et al., 1983). It has been found, however, that the colour of spores and substrate mycelia, as well as soluble pigment production, are not always taxonspecific. For this reason, as well as because determination of the colour of a pigment is, to a large extent, subjective, we used only the presence or absence of pigment production for the grouping of the Frankia strains. Subsequently, differences between strains in the colour of the pigments and in the ability to produce pigments intra- and/or extracellularly, shown in Table 2 , may be used as additional differentiating characters.

The presence or absence of pigment production in the strains studied correlated to a significant extent with their groupings on the basis of host specificity. Thus, none of the three Casuarinaceae-specific strains pro- duced pigments and most (13 of 16) of the Alnusspecific strains did not produce pigments. On the contrary, pigment production was characteristic of most (19 of 20) of the Elaeagnaceae-specific strains (Table 2).

\section{Host specificity}

As the third group of characters, our own data and literature data on host-specificity ranges of Frankia strains were used, as summarized in Table 2 . The strains tested belong to the three best-studied hostspecificity groups, Alnus, Elaeagnus and Casuarina, with some Elaeagnaceae-specific strains being able to cross-nodulate Alnus spp. The host plant of origin and the ability to nodulate within certain host-compatibility groups are known to be, to a great extent, relevant taxonomic characters (for a review, see Benson \& Silvester, 1993). Both phylogenetic (Normand et al., 1996) and DNA-DNA hybridization (Fernandez et al., 1989; Akimov \& Dobritsa, 1992) data have shown that major groupings of Frankia strains at the supraspecific level occur according to their source plant and/or host specificity.

\section{Phenotypic clusters}

Fig. 1 shows the results of cluster analysis of the standardized data on antibiotic susceptibility, pigment production and host specificity of the Frankia strains studied. Cluster groups were defined at the 1.25 distance level, while clusters were defined at the 0.74 distance level. These levels were selected after examination of the dendrogram, as they were the lowest which gave clear groupings, correlating with the hostspecificity ranges (Table 2 ) and genospecies, respectively. It should be noted, however, that the latter level is to some extent arbitrary due to missing data on genetic relatedness between members of independently delineated genospecies (Fernandez et al., 1989; Akimov \& Dobritsa, 1992; Lumini et al., 1996). Additional DNA hybridization tests may result in widening, to some extent, the limit specified and in changing the cluster structure in three cases of strain groupings occurring at levels close to this limit which are discussed below.

The majority of the Frankia strains tested (34 strains) were recovered in three cluster groups (A, E and C) which could be roughly equated with the host-specificity groups Alnus, Elaeagnus and Casuarina. The cluster groups were in turn further subdivided into eight composite and five single-member clusters. Five strains fell into five single-member clusters not included in any of the cluster groups.

Twelve strains representing three genospecies, with levels of genetic relatedness between their representatives equal to $28-30 \%$ (Akimov \& Dobritsa, 1992), fell into cluster group A. The group was subdivided 


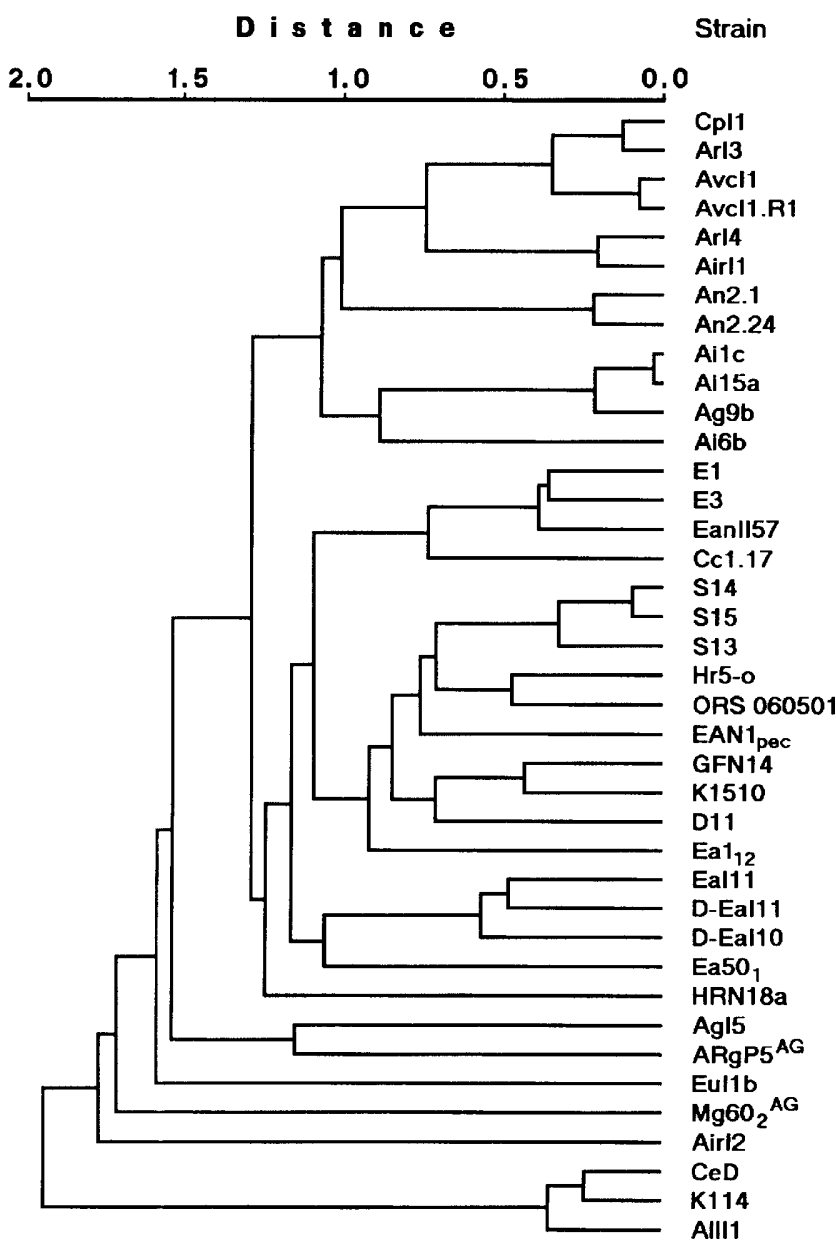

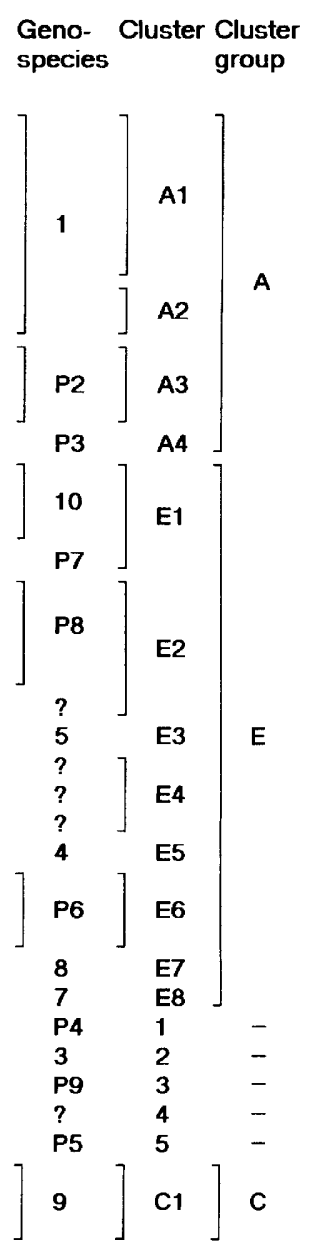

Fig. 1. UPGMA dendrogram showing the relationships among the 39 Frankia strains studied, derived from cluster analysis of the data presented in Table 2. into one single-member and three composite clusters in good agreement with the membership of the strains in the corresponding genospecies.

Eight strains of genospecies 1 (Fernandez et al., 1989; Akimov \& Dobritsa, 1992) were separated into two clusters, A1 and A2. The distance level between these (about 1.(0) significantly exceeded the minimal level observed in this study $(0 \cdot 85)$ at which representatives of different genospecies united. Thus, our data show that strains An2.1 and An2.24 (cluster A2), despite their high genomic relatedness $(81$ and $85 \%$, respectively; Akimov \& Dobritsa, 1992) to strain CpI1 (cluster A1), might deserve classification as a separate species. This does not contradict usual taxonomic practice which, in the case of inconsistency between phenotypic and DNA-DNA hybridization data, gives priority to the former (Ursing et al., 1995; Vandamme et al., 1996). It has been generally agreed that a taxonomic conclusion should be based on at least two simple tests (Bascomb et al., 1973). In this case, clear differences between strains assigned to clusters A1 and A2 were observed in the characters of pigment production and susceptibility to erythromycin, with additional differences displayed in the levels of susceptibility to chloramphenicol and streptomycin.

It is also noteworthy that the strains of cluster Al, which are not easy to differentiate by other phenotypic methods, displayed heterogeneity in their susceptibility to antibiotics. Even though some differences in inhibition zone diameters between these strains might be due to insufficient standardization of the inocula, enough differences are evident to be objective. Thus, strains ArI4 and AirI1 were distinguished from the remaining strains of the cluster by their susceptibility to clindamycin and lincomycin. Also, strain ArI4 was distinguished by a higher susceptibility to erythromycin, while strains AvcIl and AvcIl.R1 were distinguished by lower susceptibility to kanamycin.

Cluster A3 contained phenotypically similar strains of genospecies P2 (Akimov \& Dobritsa, 1992), isolated in Finland (Weber et al., 1988), which were distinguished from those in clusters $\mathrm{A} 1$ and $\mathrm{A} 2$ by their resistance to chloramphenicol and rifampicin and certain differences in their levels of susceptibility to carbenicillin, erythromycin, kanamycin and vancomycin. Strain Ai6b, the only member of genospecies P3 (Akimov \& Dobritsa, 1992), isolated in Finland (Weber et al., 1988), united with this cluster at a significant distance level (0.88). Strain Ai6b was differentiated from the strains in cluster A2 by its susceptibility to rifampicin, higher susceptibility to bacitracin, neomycin and penicillin $\mathrm{G}$ and a lower susceptibility to vancomycin. 
Cluster group E contained 19 strains separated into four composite and four single-member clusters, correlating to a significant extent with the genospecies described. It is interesting that all so-called flexible Elaeagnaceae-isolated strains, which are infective on both the Elaeagnaceae and Alnus (see Table 2), fell into cluster group $\mathrm{E}$ in accordance with their source of isolation rather than host-compatibility range. However, these did not form a homogeneous group and were recovered in three distinct clusters, E1, E6 and E8.

Cluster E1 included three phenotypically similar flexible strains of Italian origin (Margheri et al., 1985; Bosco et al., 1992) infective on both Elaeagnus and Alnus and representing genospecies 10 (Lumini et al., 1996). Another flexible strain, Cc1.17, infective on both Elaeagnaceae and Myrica (Baker, 1987) and representing single strain genospecies P7 (Akimov \& Dobritsa, 1992), fell into the same cluster at the 0.73 distance level. This distance is close to the threshold level defined for clusters and, thus, DNA hybridization is necessary to elucidate the status of this strain (genospecies). Strains of cluster El could be differentiated from all other test strains by extremely high susceptibility to novobiocin and, unlike most of the strains in cluster group E, resistance to clindamycin.

Cluster E2 included four strains of genospecies P8 (Akimov \& Dobritsa, 1992) as well as strain ORS 060501, not assigned to any genospecies (Fernandez $e t$ al., 1989). It is interesting that all Frankia strains (S14, S15 and ORS 060501) in which linear plasmids have been found (Dobritsa, 1990; S. V. Dobritsa, unpublished data) fell into the same cluster, E2. Strain EAN $1_{\text {pec }}$, a representative of genospecies 5 (Fernandez et al., 1989), was grouped to cluster E2. However, the distance level between it and the other strains, 0.76 , slightly exceeded the threshold level for clusters, chosen on the basis of DNA relatedness data. Nevertheless, the distance is close enough to the limit and therefore the status of strain $\mathrm{EAN} 1_{\text {pec }}$, assigned to a separate cluster (E3), must be defined more precisely by DNA hybridization. The clear differences in the levels of susceptibility to bacitracin, chloramphenicol, carbenicillin, kanamycin, penicillin $\mathrm{G}$ and vancomycin between strain EAN1 $1_{\text {pec }}$ and strains in cluster E2 may suggest that the status will not change.

Clusters E2 and E3 were grouped to cluster E4. The nucleus of the latter was formed by strains GFN 14 and K1510 whose genospecies affiliation and affinities are unknown, but it may be of significance that both have been isolated in China (Fernandez et al., 1989; Du \& Baker, 1992). An atypical isolate from Casuarina sp., D11, which does not nodulate members of the Casuarinaceae but is infective on Elaeagnaceae (Gauthier et al., 1981), fell into this cluster. Our data showed that strain D11 was a typical representative of the group Elaeagnus, not Casuarina, on the basis of the selection of characters. However, the 0.71 distance level between strain D11 and the other two strains of cluster E4 is close to the delimiting level for clusters. Therefore, the status and affinities of the strain need to be confirmed by DNA hybridization. Clusters E2, E3 and E4 shared their closest similarities with each other at the 0.85 distance level, but strains within clusters E2 and E4 displayed significant heterogeneity in their susceptibility to antibiotics which makes selection of characters clearly differentiating these clusters from the other clusters of cluster group $\mathrm{E}$ impossible. A singlemember cluster containing strain Eal ${ }_{12}$, representing genospecies 4 (Fernandez et al., 1989), fused to these three clusters at the 0.92 distance level. This strain was characterized by the lowest level of susceptibility to bacitracin among all test strains and by the highest level of susceptibility to erythromycin among those in cluster group E.

Three strains of Russian origin, representing genospecies P6 (Akimov \& Dobritsa, 1992; S. V. Dobritsa, unpublished data), fell into cluster E6 despite differences between them in their ability to nodulate Alnus and in pigment production (Table 2). They were distinguished from the remaining strains of cluster group $\mathrm{E}$ by the lowest levels of susceptibility to kanamycin and, in addition, they displayed the highest levels of susceptibility to bacitracin among all test strains, except EuI1b (single-member cluster 3). The latter, however, could be clearly differentiated from the strains in cluster E6 on the basis of its levels of susceptibility to ampicillin, chloramphenicol, carbenicillin, erythromycin, gentamicin, kanamycin, neomycin, novobiocin, penicillin $G$, streptomycin and tetracycline. Strain Ea $50_{1}$, the only member of genospecies 8 (Fernandez et al., 1989), was recovered in single-member cluster E7 which joined to cluster E6 at a significant distance level (1.06). It could be distinguished from the strains of cluster E6 by the colour of the pigment produced and levels of susceptibility to ampicillin, bacitracin, erythromycin, kanamycin, novobiocin, penicillin $G$ and rifampicin. In addition, strain Ea50 was the only one among all the strains tested that displayed resistance to streptomycin. Strain HRN18a, representing single-member genospecies 7 (Fernandez et al., 1989), shared little similarity with any strains of cluster group $\mathrm{E}$ and was recovered in a separate single-member cluster, E8. It displayed the highest levels of susceptibility to gentamicin and kanamycin among all test strains and the second highest levels of susceptibility to vancomycin and novobiocin.

Cluster group $\mathrm{C}$ included only one cluster, $\mathrm{Cl}$, containing three phenotypically similar members of genospecies 9 (Fernandez et al., 1989; S. V. Dobritsa, unpublished data) which could be clearly differentiated from the remainder of the strains. In addition to their ability to nodulate the Casuarinaceae, strains of this cluster were distinguished from all other test strains by their resistance to gentamicin and resistance or weak susceptibility to kanamycin, and from most strains by their resistance to the $\beta$-lactams (ampicillin, carbenicillin and penicillin $G$ ) and rifampicin. Due to these unique patterns, cluster group $\mathrm{C}$ was well separated 
from groups $\mathrm{A}$ and $\mathrm{E}$, even though phylogenetic studies (e.g. Normand et al., 1996) have revealed that Casuarina-specific strains are more closely related to Alnus-specific strains, while these two groups show less relatedness to strains in the Elaeagnus host-specificity group.

Five strains were clearly differentiated from the remainder and did not fall into any of the cluster groups. Each of them was characterized by a unique antibiogram. For instance, strain AgI5 was the only one that showed no susceptibility to tetracycline and only two strains, $\mathrm{ARgP} 5^{\mathrm{AG}}$ and AirI2, displayed resistance to vancomycin, with all other test strains being highly susceptible to this antibiotic. All five strains were recovered in single-member clusters (1-5) among which only two strains, AgI5 and ARgP5 ${ }^{\mathrm{AG}}$ (clusters 1 and 2 , respectively), joined at the $1 \cdot 15$ distance level. Strains EuIlb (Elaeagnus group), AgI5, $\mathrm{ARgP5}^{\mathrm{AG}}$ and AirI2 (Alnus group) represent single strain genospecies $\mathrm{P} 9, \mathrm{P} 4,3$ and $\mathrm{P} 5$, respectively. Their phenotypic isolation demonstrated in this study correlates with low levels of genetic relatedness (0-14\%) to representatives of the other genospecies (Fernandez et al., 1989; Akimov \& Dobritsa, 1992). Also, strain $\mathrm{ARgP5}^{\mathrm{AG}}$, the most studied of these strains, has been shown by both phylogenetic (e.g. Normand et al., 1996) and phenotypic (for a review, see Lalonde et al., 1988) studies, including isoenzyme pattern analysis (Gardes et al., 1987), to be quite distantly related to members of genospecies 1 in the Alnus infectivity group. $\mathrm{Mg} 60_{2}{ }^{\mathrm{AG}}$, the only test strain originating from the plant genus Myrica (isolated from Alnus glutinosa inoculated with crushed nodules of Myrica gale), has not been assigned to any genospecies (Fernandez et al., 1989). It was characterized in this study by the highest or closest to the highest levels of susceptibility to the $\beta$ lactams (ampicillin, carbenicillin and penicillin $G$ ) and aminoglycosides (gentamicin, kanamycin, neomycin and streptomycin), as well as to clindamycin, lincomycin, erythromycin and vancomycin.

\section{Taxonomic implications}

Analysis of the data on susceptibility to antibiotics, pigment production and host specificity resulted in a phenotypic grouping of the Frankia strains which showed good agreement with DNA hybridization data. Thus, the clusters formed may be considered to be acceptable taxa at the species level. In some cases, the characters studied clearly differentiate certain groups of strains (genospecies) and therefore could be useful for species descriptions, as discussed above for composite clusters A1, A2, A3, E1, E6 and C1. Also, each of the five strains comprising single-member clusters not in the cluster groups, as well as most of the strains comprising single-member clusters within the cluster groups, are characterized by unique combinations of characters. These peculiarities may, in principle. be used for descriptions of corresponding species. However, strains Ai6b, Ea50, HRN18a, EuI1b, AgI5, ARgP5 ${ }^{A G}$ and Airl2, recovered here in single-member clusters, all represent single-member genospecies. Description of a species represented by a single strain is considered to be inadequate (Vandamme et al., 1996), since characters of a single isolate do not reflect the phenotypic diversity of an entire species. For the same reason, additional studies are needed in the cases of genospecies 4 and 5 containing six and three strains, respectively (Fernandez et al., 1989). In this study, these were each represented by a single strain $\left(\mathrm{Ea}_{12}\right.$ and $\mathrm{EAN} 1_{\text {pec, }}$, respectively) which does not allow estimation of the phenotypic diversity of these two genospecies. Finally, for some clusters in cluster group E, containing highly diverse strains, additional phenotypic (clusters E2 and E4) and/or DNA hybridization (clusters E1 and E4) tests are needed to define corresponding species.

\section{ACKNOWLEDGEMENTS}

I wish to thank A. D. L. Akkermans, D. D. Baker, A. M. Berry, M. Bosco, M. P. Fernandez, M. P. Lechevalier, J.-S. Ruan, L. S. Sharaya, O. S. Stupar and V. Sundman, as well as the late J. G. Torrey for kindly providing Frankia strains.

\section{REFERENCES}

Akimov, V. N. \& Dobritsa, S. V. (1992). Grouping of Frankia strains on the basis of DNA relatedness. Syst Appl Microbiol 15 , $372-379$

Akkermans, A. D. L., Hahn, D. \& Baker, D. D. (1992). The family Frankiaceae. In The Prokaryotes, 2nd edn, vol. 2, pp. 1069-1084. Edited by A. Balows, H. G. Trüper, M. Dworkin, W. Harder \& K.-H. Schleifer. New York: Springer.

Baker, D. D. (1987). Relationships among pure-cultured strains of Frankia based on host specificity. Physiol Plant 70, 245-248.

Baker, D. (1990). Methods for the isolation, culture and characterization of the Frankiaceae: Soil actinomycetes and symbionts of actinorhizal plants. In Isolation of Biotechnological Organisms from Nature, pp. 213-236. Edited by D. P. Labeda. New York: McGraw-Hill.

Baker, D., Torrey, J. G. \& Kidd, G. H. (1979). Isolation by sucrosedensity fractionation and cultivation in vitro of actinomycetes from nitrogen-fixing root nodules. Nature 281, 76-78.

Baker, D., Pengelly, W. L. \& Torrey, J. G. (1981). Immunochemical analysis of relationships among isolated frankiae (Actinomycetales). Int J Syst Bacteriol 31, 148-151.

Bascomb, S., Lapage, S. P., Curtis, M. A. \& Willcox, W. R. (1973). Identification of bacteria by computer: identification of reference strains. J Gen Microbiol 77, 291-315.

Becking, J. H. (1970). Frankiaceae fam. nov. (Actinomycetales) with one new combination and six new species of the genus Frankia Brunchorst 1886, 174. Int J Syst Bacteriol 20, 201-220.

Benson, D. R. \& Silvester, W. B. (1993). Biology of Frankia strains, actinomycete symbionts of actinorhizal plants. Microbiol Rev 57, 293-319.

Berry, A. \& Torrey, J. G. (1979). Isolation and characterization in vivo and in vitro of an actinomycetous endophyte from Alnus rubra Bong. In Symbiotic Nitrogen Fixation in the Management of Temperate Forests, pp. 69-83. Edited by J. C. Gordon, C. T. Wheeler \& D. A. Perry. Corvallis, OR: Oregon State University Press.

Blom, J., Roelofsen, W. \& Akkermans, A. D. L. (1980). Growth of 
Frankia Avcll on media containing Tween 80 as C-source. FEMS Microbiol Lett 9, 131-135.

Bosco, M., Fernandez, M. P., Simonet, P., Materassi, R. \& Normand, P. (1992). Evidence that some Frankia sp. strains are able to cross boundaries between Alnus and Elaeagnus host specificity groups. Appl Environ Microbiol 58, 1569-1576.

Burggraaf, A. J. P. \& Shipton, W. A. (1982). Estimates of Frankia growth under various $\mathrm{pH}$ and temperature regimes. Plant Soil 69, 135-147.

Callaham, D., Del Tredici, P. \& Torrey, J. G. (1978). Isolation and cultivation in vitro of the actinomycete causing root nodulation in Comptonia. Science 199, 899-902.

Carrasco, A., Salyards, J. R. \& Berry, A. M. (1995). Studies of two Frankia strains isolated from Trevoa trinervis Miers. Plant Soil 171, 359-363.

Carú, M. (1993). Characterization of native Frankia strains isolated from chilean shrubs (Rhamnaceae). Plant Soil 157, $137-145$

Dawson, J. O. \& Gibson, A. H. (1987). Sensitivity of selected Frankia isolates from Casuarina, Allocasuarina and North American host plants to sodium chloride. Physiol Plant 70, 272-278.

Diem, H. G. \& Dommergues, Y. (1983). The isolation of Frankia from nodules of Casuarina. Can J Bot 61, 2822-2825.

Dobritsa, S. v. (1990). A linear plasmid in two Frankia strains. Abstracts of the 8th International Congress on Nitrogen Fixation, Knoxville, TN, USA. Abstract E-85.

Dobritsa, S. V. \& Stupar, O. S. (1989). Genetic heterogeneity among Frankia isolates from root nodules of individual actinorhizal plants. FEMS Microbiol Lett 58, 287-292.

Dobritsa, S. V., Novik, S. N. \& Stupar, O. S. (1990). Infectivity and host specificity of strains of Frankia. Microbiology (English translation of Mikrobiologiya) 59, 210-214.

Du, D. \& Baker, D. D. (1992). Actinorhizal host-specificity of Chinese Frankia strains. Plant Soil 144, 113-116.

Faure-Raynaud, M., Daniere, C., Moiroud, A. \& Capellano, A. (1990). Preliminary characterization of an ineffective Frankia derived from a spontaneously neomycin-resistant strain. Plant Soil 129, 165-172.

Fernandez, M. P., Meugnier, H., Grimont, P. A. D. \& Bardin, R. (1989). Deoxyribonucleic acid relatedness among members of the genus Frankia. Int J Syst Bacteriol 39, 424-429.

Friedman, R. \& MacLowry, J. (1973). Computer identification of bacteria on the basis of their antibiotic susceptibility patterns. Appl Microbiol 26, 314-317.

Gardes, M., Bousquet, J. \& Lalonde, M. (1987). Isozyme variation among 40 Frankia strains. Appl Environ Microbiol 53, 1596-1603.

Gauthier, D., Diem, H. G. \& Dommergues, Y. (1981). Infectivité et effectivité de souches de Frankia isolées de nodules de Casuarina equisetifolia et d'Hippophaë rhamnoides. C R Acad Sci Paris Ser III 293, 489-491.

Gauthier, D., Frioni, L., Diem, H. G. \& Dommergues, Y. (1984). The Colletia spinosissima-Frankia symbiosis. Acta Oecol 5, 231-239.

Gilardi, G. L. (1971). Antimicrobial susceptibility as a diagnostic aid in the identification of nonfermenting gram-negative bacteria. Appl Microbiol 22, 821-823.

Goodfellow, M. \& Orchard, V. A. (1974). Antibiotic sensitivity of some nocardioform bacteria and its value as a criterion for taxonomy. J Gen Microbiol 83, 375-387.

Gordon, R. E. \& Barnett, D. A. (1977). Resistance to rifampin and lysozyme of strains of some species of Mycobacterium and Nocardia as a taxonomic tool. Int J Syst Bacteriol 27, 176-178.

Hafeez, F., Akkermans, A. D. L. \& Chaudhary, A. H. (1984). Morphology, physiology and infectivity of two Frankia isolates An 1 and An 2 from root nodules of Alnus nitida. Plant Soil 78, 45-59.

Hahn, D., Dorsch, M., Stackebrandt, E. \& Akkermans, A. D. L. (1989). Synthetic oligonucleotide probes for identification of Frankia strains. Plant Soil 118, 211-219.

Lalonde, M., Calvert, H. E. \& Pine, S. (1981). Isolation and use of Frankia strains in actinorhizae formation. In Current Perspectives in Nitrogen Fixation, pp. 296-299. Edited by A. H. Gibson \& W. E. Newton. Canberra: Australian Academy of Sciences.

Lalonde, M., Simon, L., Bousquet, J. \& Séguin, A. (1988). Advances in the taxonomy of Frankia: recognition of species alni and elaeagni and novel subspecies pommerii and vandijkii. In Nitrogen Fixation: Hundred Years After, pp. 671-680. Edited by H. Bothe, F. J. de Bruijn \& W. E. Newton. New York: Gustav Fisher.

Lechevalier, M. P. (1994). Taxonomy of the genus Frankia (Actinomycetales). Int $J$ Syst Bacteriol 44, 1-8.

Lechevalier, M. P., Horrière, F. \& Lechevalier, H. A. (1982). The biology of Frankia and related organisms. Dev Ind Microbiol 23, 51-60.

Lechevalier, M. P., Baker, D. \& Horrière, F. (1983). Physiology, chemistry, serology and infectivity of two Frankia isolates from Alnus incana subsp. rugosa. Can J Bot 61, 2826-2833.

Lumini, E., Bosco, M. \& Fernandez, M. P. (1996). PCR-RFLP and total DNA homology revealed three related genomic species among broad-host-range Frankia strains. FEMS Microbiol Ecol 21, 303-311.

Margheri, M. C., Vagnoli, L., Favilli, F. \& Sili, C. (1985). Proprietà morfo-fisiologiche di Frankia ceppo EanII57 da Elaeagnus angustifolia, infettivo su Alnus glutinosa. Ann Microbiol 35, 143-153.

Mirza, M. S., Janse, J. D., Hahn, D. \& Akkermans, A. D. L. (1991). Identification of atypical Frankia strains by fatty acid analysis. FEMS Microbiol Lett 83, 91-98.

Moiroud, A. \& Faure-Raynaud, M. (1983). Influence de quelques herbicides à large spectre sur la croissance et l'infectivité de cultures pures de Frankia. Plant Soil 74, 133-136.

Murry, M. A., Fontaine, M. S. \& Torrey, J. G. (1984). Growth kinetics and nitrogenase induction in Frankia sp. HFPArI3 grown in batch culture. Plant Soil 78, 61-78.

Naik, S. P., Samsonoff, W. A. \& Ruck, R. E. (1989). Effects of surface-active agents on drug susceptibility levels and ultrastructure of Mycobacterium avium complex organisms isolated from AIDS patients. Diagn Microbiol Infect Dis 11, 11-19.

Nazaret, S., Simonet, P., Normand, P. \& Bardin, R. (1989). Genetic diversity among Frankia isolated from Casuarina nodules. Plant Soil 118, 241-247.

Nikitin, D. I., Vishnevetskaya, O. Yu. \& Zlatkin, I. V. (1988). Grouping of oligotrophic microorganisms on the basis of their antibiotic resistance and the dynamic membrane characteristics. Microbiology (English translation of Mikrobiologiya) 57, 210-215.

Normand, P. \& Lalonde, M. (1982). Evaluation of Frankia strains isolated from provenances of two Alnus species. Can J Microbiol 28, 1133-1142.

Normand, P. \& Lalonde, M. (1986). The genetics of actinorhizal Frankia: a review. Plant Soil 90, 429-453. 
Normand, P., Orso, S., Cournoyer, B., Jeannin, P., Chapelon, C., Dawson, J., Evtushenko, L. \& Misra, A. K. (1996). Molecular phylogeny of the genus Frankia and related genera and emendation of the family Frankiaceae. Int J Syst Bacteriol 46, $1-9$.

Penner, J. L. \& Preston, M. A. (1980). Differences among Providencia species in their in vitro susceptibilities to five antibiotics. Antimicrob Agents Chemother 18, 868-871.

Petralli, J., Russell, E., Kataoka, A. \& Merigan, T. C. (1970). Online computer quality control of antibiotic-sensitivity testing. New Engl J Med 283, 735-738.

Reva, O. N., Vyunitskaya, V. A., Reznik, S. R., Kozachko, I. A. \& Smirnov, V. V. (1995). Antibiotic susceptibility as a taxonomic characteristic of the genus Bacillus. Int $J$ Syst Bacteriol 45, 409-411.

Scavizzi, M. \& Bronner, F. (1987). Un modèle statistique d'interprétation de l'antibiogramme. C R Acad Sci Paris Ser III 304, 325-329.

Sharaya, L. S., Parijskaya, A. N. \& Kalakoutskii, L. V. (1985). Isolation of pure cultures of actinomycetes of the genus Frankia from nitrogen-fixing root nodules of silver oleaster and sea buckthorn. Mikrobiologiya 54, 1017-1018 (in Russian).

Shi, Y., Ruan, J., Zakrzewska-Czerwinska, J. \& Mordarski, M. (1991). DNA homology of some Frankia strains. Actinomycetes 2, 86-87.

Shipton, W. A. \& Burggraaf, A. J. P. (1982a). A comparison of the requirements for various carbon and nitrogen sources and vitamins in some Frankia isolates. Plant Soil 69, 149-161.

Shipton, W. A. \& Burggraaf, A. J. P. (1982b). Frankia growth and activity as influenced by water potential. Plant Soil 69, 293-297.

Sielaff, B. H., Johnson, E. A. \& Matsen, J. M. (1976). Computerassisted bacterial identification utilizing antimicrobial susceptibility profiles generated by Autobac 1. J Clin Microbiol 3, 105-109.

Simon, L., Jabaji-Hare, S., Bousquet, J. \& Lalonde, M. (1989). Confirmation of Frankia species using cellular fatty acid analysis. Sist Appl Microbiol 11, 229-235.

Simonet, P., Normand, P., Moiroud, A. \& Lalonde, M. (1985). Restriction enzyme digestion patterns of Frankia plasmids. Plant Soil 87, 49-60.

Sneath, P. H. A. \& Sokal, R. R. (1973). Numerical Taxonomy. The Principles and Practice of Numerical Classification. San Francisco, CA: W. H. Freeman.
St-Laurent, L., Bousquet, J., Simon, L. \& Lalonde, M. (1987). Separation of various Frankia strains in the Alnus and Elaeagnus host specificity groups using sugar analysis. Can J Microbiol 33, 764-772.

Torrey, J. G. (1990). Cross-inoculation groups within Frankia and host-endosymbiont associations. In The Biology of Frankia and Actinorhizal Plants, pp. 83-106. Edited by C. R. Schwintzer \& J. D. Tjepkema. San Diego, CA: Academic Press.

Tsukamura, M. (1981). Test for susceptibility to mitomycin C as aids for differentiating the genus Rhodococcus from the genus Nocardia and for differentiating Mycobacterium fortuitum and Mycobacterium chelonei from other rapidly growing mycobacteria. Microbiol Immunol 25, 1197-1199.

Tsukamura, M. (1982). Differentiation between the genera Rhodococcus and Nocardia and between species of the genus Mycobacterium by susceptibility to bleomycin. J Gen Microbiol 128, 2385-2388.

Tsukamura, M. (1988). Differentiation between genera Rhodococcus and Nocardia by susceptibility testing to kanamycin and some other antituberculosis agents. Microbiol Immunol 32, $441-445$.

Ursing, J. B., Rosselló-Mora, R. A., Garcia-Valdés, E. \& Lalucat, J. (1995). Taxonomic note: a pragmatic approach to the nomenclature of phenotypically similar genomic groups. Int $J$ Syst Bacteriol 45, 604.

Vandamme, P., Pot, B., Gillis, M., De Vos, P., Kersters, K. \& Swings, J. (1996). Polyphasic taxonomy, a consensus approach to bacterial systematics. Microbiol Rev 60, 407-438.

Vogel, C. S. \& Dawson, J. O. (1986). In vitro growth of five Frankia isolates in the presence of four phenolic acids and juglone. Soil Biol Biochem 18, 227-231.

Wayne, L. G., Brenner, D. J., Colwell, R. R. \& 9 other authors (1987). International Committee on Systematic Bacteriology. Report of the ad hoc committee on reconciliation of approaches to bacterial systematics. Int J Syst Bacteriol 37, 463-464.

Weber, A., Smolander, A., Nurmiaho-Lassila, E.-L. \& Sundman, V. (1988). Isolation and characterization of Frankia strains from Alnus incana and Alnus glutinosa in Finland. Symbiosis $\mathbf{6}$, 97-116.

Williams, S. T., Goodfellow, M., Alderson, G., Wellington, E. M. H., Sneath, P. H. A. \& Sackin, M. J. (1983). Numerical classification of Streptomyces and related genera. $J$ Gen Microbiol 129, 1743-1813.

Zhang, Z. \& Torrey, J. G. (1985). Studies of an effective strain of Frankia from Allocasuarina. Plant Soil 87, 1-16. 
